\title{
Inter-tumor heterogeneity of PD-L1 expression in non-small cell lung cancer
}

\author{
Yuichi Saito ${ }^{1,2}$, Sho Horiuchi ${ }^{1}$, Hiroaki Morooka ${ }^{1}$, Takayuki Ibi ${ }^{1}$, Nobumasa Takahashi ${ }^{1}$, Tomohiko Ikeya ${ }^{1}$, \\ Yoshihiko Shimizu $^{3}$, Eishin Hoshi ${ }^{1}$ \\ ${ }^{1}$ Department of Thoracic Surgery, Saitama Cardiovascular and Respiratory Center, Saitama, Japan; ${ }^{2}$ Department of Surgery, Teikyo University \\ School of Medicine, Tokyo, Japan; ${ }^{3}$ Department of Pathology, Saitama Cardiovascular and Respiratory Center, Saitama, Japan \\ Contributions: (I) Conception and design: Y Saito; (II) Administrative support: None; (III) Provision of study materials or patients: Y Shimizu; (IV) \\ Collection and assembly of data: All authors; (V) Data analysis and interpretation: All authors; (VI) Manuscript writing: All authors; (VII) Final \\ approval of manuscript: All authors. \\ Correspondence to: Yuichi Saito. Department of Thoracic Surgery, Saitama Cardiovascular and Respiratory Center, 1696 Itai, Kumagaya, Saitama 360- \\ 0197, Japan. Email: k3699004@gmail.com.
}

Background: The Dako PD-L1 immunohistochemistry (IHC) 22C3 pharmDx and the Dako 28-8 IHC pharmDx assays were approved by the US Food and Drug Administration, as a companion diagnostic test for pembrolizumab (Keytruda, Merk, Kenilworth, NJ, USA) and a complementary diagnostic test for nivolumab (Opdivo, Bristol Meyer Squibb, New York, NY, USA) in non-small cell lung cancer (NSCLC), respectively. Increased PD-L1 expression levels can be associated with greater therapeutic efficacy of pembrolizumab relative to other anti-PD-1 agents. However, in treatment decision making, little is known about which tissue (primary or metastatic lesion) should be stained by 22C3 antibody. We investigated the relationship between PD-L1 expression in primary tumors and paired metastatic lymph nodes using the 22C3 assay, and evaluated the concordance between the $22 \mathrm{C} 3$ and $28-8$ assays.

Methods: PD-L1 expression was evaluated in cells from primary tumors and paired metastatic lymph nodes using the 22C3 and 28-8 IHC assays. Total 35 patients with primary tumor and paired metastatic lymph node were enrolled into this study, and all samples were surgically resected, formalin-fixed, and paraffinembedded NSCLC tissues. Tumor cells exhibiting complete or partial membrane staining, were considered as PD-L1 positive. On the basis of tumor proportion score (TPS), all samples were classified as no expression (TPS: <1\%), low expression (TPS: 1-49\%), or high expression (TPS: $\geq 50 \%$ ).

Results: TPS distribution was markedly different between primary tumors and paired metastatic lymph nodes. In 22C3 IHC assay, TPS similar to that of metastatic lymph nodes was demonstrated in 10 primary tumors, and concordance rate between them was $28.6 \%$. Concurrently, in $28-8$ IHC assay, 11 primary tumors had TPS similar to that of metastatic lymph nodes, with a concordance rate of $31.4 \%$.

Conclusions: TPS concordance rates (for both 22C3 and 28-8 antibodies) between primary tumors and paired lymph nodes were low. Inter-tumor heterogeneity of PD-L1 expression is an important issue for clinical oncologists during treatment planning.

Keywords: Lung cancer; heterogeneity; immunotherapy; programmed death-ligand 1 (PD-L1); tumor proportion score (TPS)

Submitted Aug 18, 2019. Accepted for publication Nov 12, 2019.

doi: $10.21037 /$ jtd.2019.12.24

View this article at: http://dx.doi.org/10.21037/jtd.2019.12.24 


\section{Introduction}

Lung cancer is the leading cause of cancer-related mortality worldwide (1-3). However, there have been no marked improvements in the long-term survival of patients with advanced non-small cell lung cancer (NSCLC) over the past couple of decades (4). Nonetheless, following advances in research on immune checkpoint mechanisms (5-9), programmed death 1 (PD-1) or programmed death-ligand 1 (PD-L1)-targeted therapies have been rapidly developed, and several clinical trials have shown their antitumor effects with remarkable responses in patients with NSCLC (10-13). Based on results of these clinical trials, some immune checkpoint inhibitors have been recommended as standard treatments according to the therapeutic algorithm included in the current guidelines for primary lung cancer treatment (14-16) Nowadays, some monoclonal antibodies (pembrolizumab, nivolumab, and atezolizumab) are available in clinical practice for use as second-line treatments in patients with both squamous and nonsquamous NSCLC. However, only pembrolizumab can be used as the first-line treatment in patients exhibiting high PD-L1 expression (17).

At the time of approval by the Food and Drug Administration (FDA), the $22 \mathrm{C} 3$ antibody clone used in the PD-L1 immunohistochemistry (IHC) assay was approved as a companion diagnostic antibody for pembrolizumab, whereas the 28-8 clone was approved as a complementary diagnostic antibody for nivolumab and atezolizumab to determine the eligibility of patients with NSCLC $(18,19)$. The Blueprint Project Team has recently demonstrated that all 3 PD-L1 IHC assays (22C3, 28-8, and SP263) are interchangeable; thus, use of any of these is anticipated as a companion or complementary diagnostic test in the future (20). Furthermore, other research groups have investigated and reported on the PD-L1 staining heterogeneity shown between large and small specimens from the same tumor (intra-tumor heterogeneity) (21-23). However, there have also been a few reports regarding the heterogeneity observed between primary and metastatic tumors in the same patient (inter-tumor heterogeneity).

In this study, we aimed to explore inter-tumor heterogeneity between primary and secondary tumors (paired metastatic lymph nodes) obtained from the same patient with NSCLC using PD-L1 IHC 22C3 and 28-8 antibodies (Dako, Glostrup, Denmark). Moreover, we assessed the interchangeability of $22 \mathrm{C} 3$ and 28-8 IHC staining in both primary tumors and lymph nodes.

\section{Methods}

\section{Subjects and tumor samples}

Between December 1, 2014 and May 7, 2018, 517 patients with NSCLC underwent surgical resection at the Saitama Cardiovascular and Respiratory Center, including 45 (8.5\%) pathological N1 tumors, $50(9.5 \%) \mathrm{N} 2$ tumors, and $1(0.18 \%) \mathrm{N} 3$ tumor. In this study, the inclusion criteria were that (I) surgery cases with lymph node dissections, (II) histologically NSCLC, (III) pathologically nodal positive, and (IV) informed consent. However, we excluded all cases with insufficient volume for PD-L1 testing, and did not include any biopsy samples. Finally, only 35 (6.8\%) were available for immunohistochemical staining using the PD-L1 22C3 and 28-8 antibodies. Pathological diagnosis and tumor proportion score (TPS) were determined by an expert pathologist (Y Shimizu) based on the WHO classification version 8 and IASLC Atlas of PD-L1 Testing in Lung Cancer $(24,25)$. Written informed consent for medical research was obtained from all patients. Clinical and pathological data were stored in a database in accordance with hospital confidentiality and privacy rules. The study protocol was approved by the institutional review board of Saitama Cardiovascular and Respiratory Center (2018005).

\section{PD-L1 IHC using the 22C3 and 28-8 antibodies}

PD-L1 IHC 22C3 and 28-8 pharmDx antibodies (Dako, Glostrup, Denmark) were used in this study. The assays were performed using the Dako Autostainer Link 48 system (Dako, Glostrup, Denmark) employing an automated staining protocol $(26,27)$. According to the manufacturer's instructions, all block specimens of formalin-fixed, paraffinembedded tissues were sectioned at a thickness of 4-5 $\mu \mathrm{m}$. After deparaffinization, sections were incubated for $20 \mathrm{~min}$ at $97{ }^{\circ} \mathrm{C}$ in the Autostainer slide rack, and then stored in EnVision FLEX Wash Buffer (Dako, Glostrup, Denmark) for $5 \mathrm{~min}$ at room temperature. Finally, according to each manufactures' instructions, all staining and counterstaining were performed along with a positive control and a negative antibody control (buffer, no primary antibody).

\section{TPS}

PD-L1 expression was evaluated only in tumor cells from primary tumors and paired metastatic lymph nodes (Figure 1). A tumor cell was defined as positive for PD-L1 staining, 


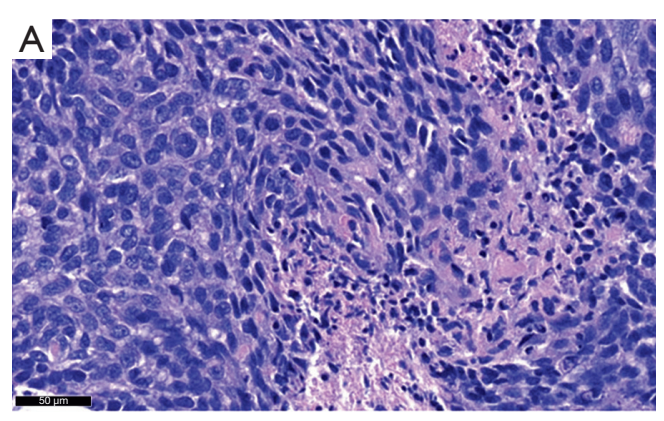

HE

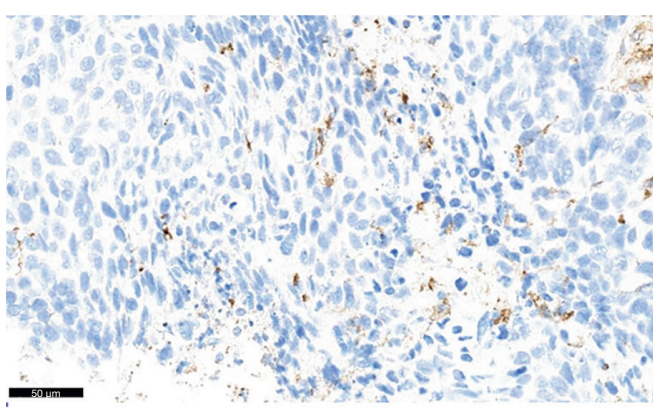

TPS (22C3): $<1 \%$

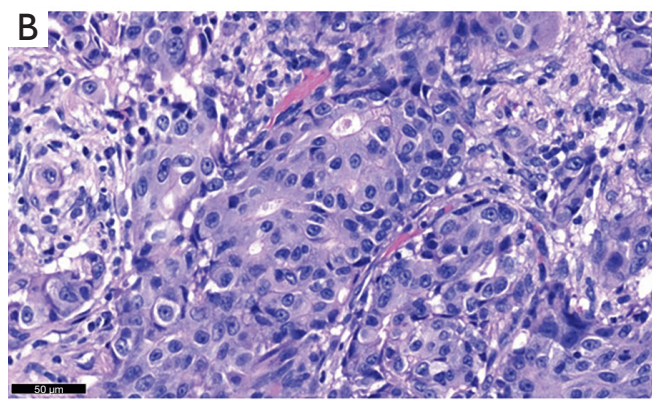

HE

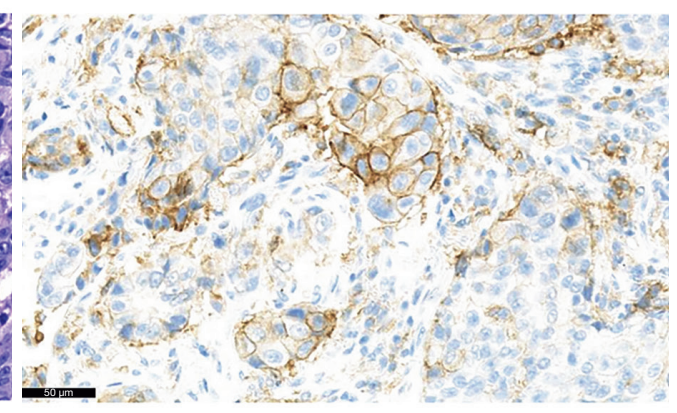

TPS (22C3): 1-49\%

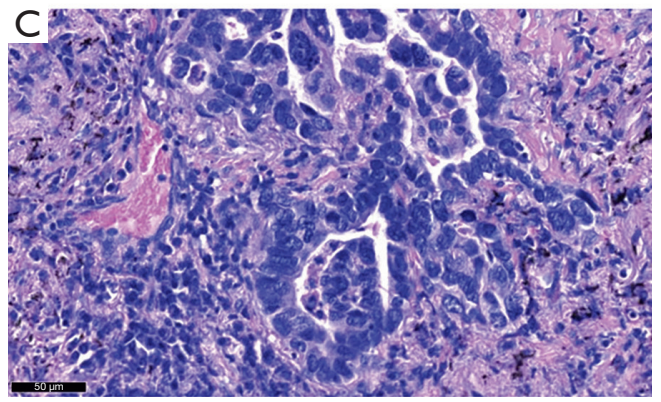

$\mathrm{HE}$

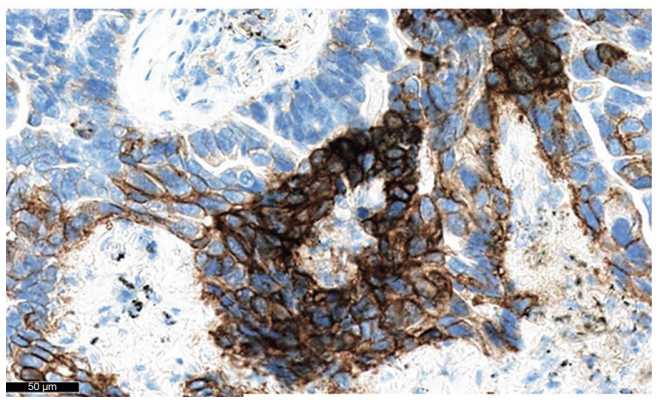

TPS (22C3): $>50 \%$

Figure 1 Scoring system of PD-L1 22C3 immunohistochemistry assay. At least 100 viable tumor cells were evaluated to judge PD-L1 immunohistochemistry positivity. PD-L1 positivity was defined as staining of the tumor cell membrane excluding cytoplasmic staining. All slides were evaluated at magnifications of $10 \times, 20 \times$, and $40 \times$. Each representative image demonstrated $<1 \%$ positivity (A), 1-49\% positivity (B), and $>50 \%$ positivity (C). TPS, tumor proportion score.

whenever any partial or complete membranous staining was detected (note: limited cytoplasmic only staining did not indicate positive staining). At least 100 viable tumor cells were required to judge PD-L1 IHC positivity on a slide. The pathologist evaluated all slides at $10 \times, 20 \times$, and $40 \times$ magnifications. TPS was defined as the percentage of positive viable tumor cells among all viable tumor cells evaluated $(\mathrm{TPS}=$ positive tumor cells/positive plus negative tumor cells). According to TPS expression levels,
3 subgroups were created: (I) no expression $(<1 \%)$, (II) low expression (1-49\%), and (III) high expression ( $\geq 50 \%)$.

\section{Statistical analysis}

All statistical analyses were conducted using SPSS 19.0 software (SPSS, Chicago, IL, USA), and a P value of $<0.05$ was considered significant. Pearson's correlation was used to evaluate the correlation coefficient between primary tumors 
Table 1 Clinical characteristics of study patients

\begin{tabular}{lc}
\hline Characteristic & $\mathrm{N}(\%)$ \\
\hline Age, years & $66.8 \pm 9.4$ \\
Gender & \\
Male & $25(71.4)$ \\
Female & $10(28.6)$ \\
Smoking status & \\
Smoker & $25(71.4)$ \\
Never-smokers & $10(28.6)$ \\
Histology & \\
Adenocarcinoma & $27(77.1)$ \\
Squamous cell carcinoma & $6(17.1)$ \\
Other & $2(5.7)$ \\
Invasive size & $11(31.4)$ \\
$\leq 30$ mm & $23(65.7)$ \\
>30 mm & $1(2.9)$ \\
Nodal status & \\
pN1 & $24(68.6)$ \\
PN2/pN3 & $11(31.4)$ \\
Negitive mutation &
\end{tabular}

Data on age: mean \pm SD. SD, standard deviation.

and paired metastatic lymph nodes, and between the 22C3 and 28-8 antibodies.

\section{Results}

\section{Patient characteristics}

Mean age of the 35 patients was 66.8 years ( $43-83$ years) (Table 1). The majority of patients were male $(\mathrm{n}=25$, $71.4 \%)$, and 25 were smokers (71.4\%). Of the 35 patients,
Table 2 Concordant rate of tumor proportion score between primary tumor and paired metastatic lymph node

\begin{tabular}{lc}
\hline Assay & Rate of concordance \\
\hline Dako 22C3 & $28.6 \%$ \\
Dako 28-8 & $31.4 \%$ \\
\hline
\end{tabular}

$27(77.1 \%)$ were diagnosed with adenocarcinoma and 6 (17.1\%) with squamous cell carcinoma. In total, ten patients exhibited pathological N1 tumors (28.6\%), whereas the remaining patients featured $\mathrm{N} 2$ or $\mathrm{N} 3$ tumors. Overall, $11(31.4 \%)$ tumors were epidermal growth factor receptor (EGFR) mutation-positive, and only 1 (2.9\%) tumor was anaplastic lymphoma kinase (ALK) fusion gene-positive and EGFR mutation-negative. TPS for both primary tumors and metastatic lymph nodes is presented in Table 2 . Although the subgroup distribution for TPS was similar between the 22C3 and 28-8 antibodies, there were differences exhibited between primary tumors and paired lymph nodes $(\mathrm{P}<0.001$ and $\mathrm{P}<0.001$, respectively).

\section{TPS for Dako 22C3 and 28-8 pharmDx}

The distribution of TPS categories was very similar between the 22C 3 and 28-8 antibodies in both primary tumors (Figure $2 A, B$ ) and metastatic lymph nodes (Figure 2C,D). Conversely, TPS values were different between primary tumors and lymph nodes in both the 22C3 (Figure 2A,C) and 28-8 assays (Figure 2B,D). Table 2 demonstrated each concordant rate of TPS between primary tumor and lymph node. TPS values in primary tumors using the $22 \mathrm{C} 3$ and $28-8$ antibodies were about $20 \%$ in the no expression, $60 \%$ in the low expression, and $20 \%$ in the high expression groups. In contrast, in metastatic lymph nodes, proportion score values were approximately $50 \%$ in the no expression, $35 \%$ in the low expression, and $15 \%$ in the high expression groups.

\section{Interrelationship of TPS between Dako 22C3 and 28-8 pharmDx}

In the PD-L1 IHC 22C3 assay, 26 primary tumors showed the same TPS as that of their paired metastatic lymph nodes (Table 3), classifying 5 tumors (14.3\%) in the no expression, 17 tumors (48.6\%) in the low expression, and 4 tumors $(11.4 \%)$ in the high expression groups. Conversely, in the 

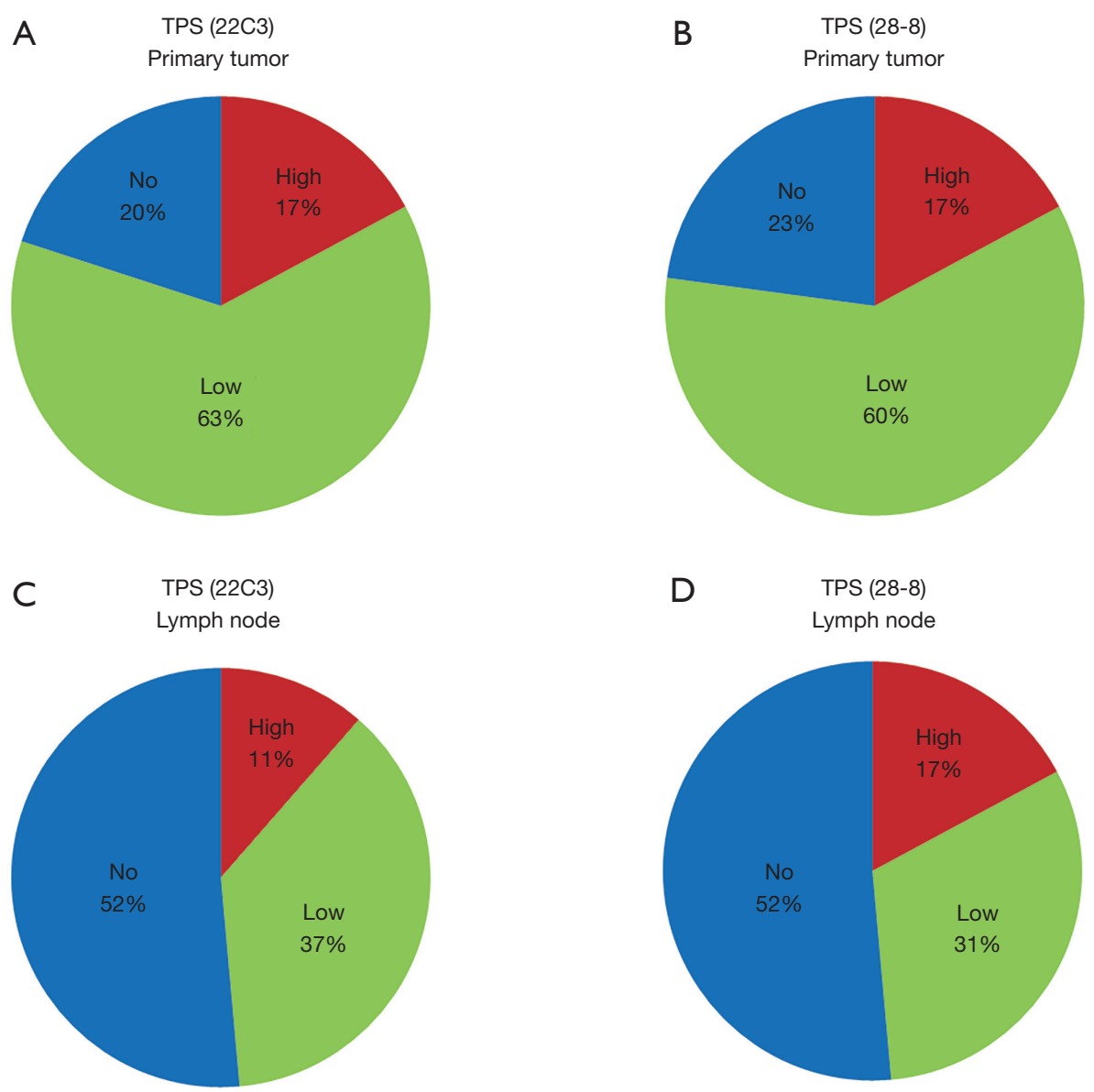

Figure 2 Tumor proportion score (TPS) of primary tumors and their paired metastatic lymph nodes. All cases were classified into 3 groups by PD-L1 expression using the Dako PD-L1 IHC 22C3 or 28-8 pharmDx assays; high expression ( $\geq 50 \%)$, low expression (1-49\%), and no expression $(<1 \%)$ are represented in red, green, and blue, respectively. (A) Pie chart representing TPS categories in primary tumors stained using the 22C3 antibody; (B) pie chart representing TPS categories in primary tumors stained with the 28-8 antibody. Primary tumors demonstrated similar TPS values in both assays; (C) pie chart representing TPS categories in metastatic lymph nodes using the 22C3 antibody; (D) last pie chart representing TPS categories in metastatic lymph nodes using the 28-8 IHC antibody. Both assays resulted in similar TPS percentages between primary tumors and lymph nodes. However, there was no similarity observed in TPS values between primary tumors and paired lymph nodes for both $22 \mathrm{C} 3$ and 28-8 IHC assays.

Table 3 Tumor proportion scores of primary tumors by Dako PDL1 IHC 22C3 PharmDx, and Dako PD-L1 IHC 28-8 PharmDx assays

\begin{tabular}{llcl}
\hline \multirow{2}{*}{ Dako 22C3 } & \multicolumn{3}{c}{ Dako 28-8 (\%) } \\
\cline { 2 - 4 } & \multicolumn{1}{c}{ No } & Low & High \\
\hline No expression $(<1 \%)$ & $5(14.3)$ & $2(5.7)$ & $0(0)$ \\
Low expression (1-49\%) & $3(8.6)$ & $17(48.6)$ & $2(5.7)$ \\
High expression $(>50 \%)$ & $0(0)$ & $2(5.7)$ & $4(11.4)$ \\
\hline
\end{tabular}

Pearson's chi-square test: $\mathrm{P}<0.001$.
PD-L1 IHC 28-8 assay, 25 primary tumors showed the same TPS as that of their paired metastatic lymph nodes, classifying 14 tumors (40.0\%) in the no expression, 7 tumors $(20.0 \%)$ in the low expression, and 4 tumors $(11.4 \%)$ in the high expression groups (Table 4). TPS concordance rates were $74.2 \%$ (primary tumors) and $71.4 \%$ (lymph nodes) between the two assays (22C 3 and 28-8 antibodies). In addition, Pearson's chi-square test revealed a significant difference between the performed assays $(\mathrm{P}<0.001$ for $22 \mathrm{C} 3$ and $\mathrm{P}<0.001$ for $28-8)$. 
Table 4 Tumor proportion scores of lymph nodes by Dako PD-L1 IHC 22C3 PharmDx, and Dako PD-L1 IHC 28-8 PharmDx assays

\begin{tabular}{lccl}
\hline \multirow{2}{*}{ Dako 22C3 } & \multicolumn{3}{c}{ Dako 28-8 (\%) } \\
\cline { 2 - 4 } & \multicolumn{1}{c}{ No } & \multicolumn{1}{c}{ Low } & High \\
\hline No expression $(<1 \%)$ & $14(40.0)$ & $4(11.4)$ & $0(0)$ \\
Low expression (1-49\%) & $4(11.4)$ & $7(20.0)$ & $2(5.7)$ \\
High expression (>50\%) & $0(0)$ & $0(0)$ & $4(11.4)$ \\
\hline
\end{tabular}

Pearson's chi-square test: $\mathrm{P}<0.001$.

Table 5 Tumor proportion scores of primary tumors and lymph nodes by Dako PD-L1 IHC 22C3 PharmDx

\begin{tabular}{lccl}
\hline \multirow{2}{*}{ Primary tumor } & \multicolumn{3}{c}{ Lymph node (\%) } \\
\cline { 2 - 4 } & No & Low & High \\
\hline No expression $(<1 \%)$ & $4(11.4)$ & $3(8.6)$ & $0(0)$ \\
Low expression (1-49\%) & $12(34.3)$ & $6(17.1)$ & $4(11.4)$ \\
High expression (>50\%) & $2(5.7)$ & $4(11.4)$ & $0(0)$ \\
\hline
\end{tabular}

Pearson's chi-square test: $P=0.300$.

Table 6 Tumor proportion scores of primary tumors and lymph nodes by Dako PD-L1 IHC 28-8 PharmDx assay

\begin{tabular}{lccl}
\hline \multirow{2}{*}{ Primary tumor } & \multicolumn{3}{c}{ Lymph node (\%) } \\
\cline { 2 - 4 } & No & Low & High \\
\hline No expression $(<1 \%)$ & $4(11.4)$ & $3(8.6)$ & $1(2.9)$ \\
Low expression (1-49\%) & $11(31.4)$ & $6(17.1)$ & $4(11.4)$ \\
High expression $(>50 \%)$ & $3(8.6)$ & $2(5.7)$ & $1(2.9)$ \\
\hline
\end{tabular}

Pearson's chi-square test: $P=0.989$.

\section{Interrelationship of TPS between primary tumors and lymph nodes}

When we performed the PD-L1 IHC 22C3 assay, ten primary tumors showed the same TPS as that of their paired metastatic lymph nodes, classifying $4(11.4 \%)$ in the no expression and 6 $(17.1 \%)$ in the low expression groups (Table 5). Surprisingly, there was no case categorized in the high expression group for neither the ten primary tumors nor their paired metastatic lymph nodes. The concordance rate between the ten primary tumors and their paired lymph nodes was $28.6 \%$, and the chisquare test revealed no significant differences between the performed assays $(\mathrm{P}=0.300)$.

Concurrently, when the PD-L1 IHC 28-8 assay was performed, 11 primary tumors showed the same TPS as that of their paired metastatic lymph nodes, classifying 4 (11.4\%) in the no expression, $6(17.1 \%)$ in the low expression, and $1(2.9 \%)$ in the high expression groups (Table 6). The calculated concordance rate was $31.4 \%(\mathrm{P}=0.989)$.

\section{Interrelationship of PD-L1 expression}

The percentage of PD-L1 positive stains for each case is shown in Figure 3A. Regarding PD-L1 expression in primary tumors, the fitting curves of both scatter plots for the two distinct PD-L1 assays were similar, exhibiting a high correlation coefficient (Figure 3B). In the case of the metastatic lymph nodes, very similar fitting curves and a high correlation coefficient were also observed (Figure 3C). Conversely, PD-L1 expression distribution among tumor tissues was rather different, indicating a discordance between primary tumors and paired metastatic lymph nodes for both the 22C3 and the 28-8 IHC assays (Figure 3D,E).

\section{Discussion}

In recent years, tumor profiling by genotyping and IHC along with histological diagnosis of primary lung cancer, which have already become common in clinical practice, have led to a dramatic increase in the roles of pulmonary pathologists. According to the National Comprehensive Cancer Network guidelines (15), NSCLC is classified based on genomic alterations [in EGFR, $A L K$, ROS protooncogene 1 (ROS1), and B-Raf proto-oncogene (BRAF)] or immunological markers (PD-L1 IHC). Based on results of a phase III investigation, pembrolizumab was approved for use in patients with tumors showing PD-L1 expression $>50 \%$ (11), and the PD-L1 IHC 22C3 (Dako) assay was approved by both the US FDA and the European Medicines Agency as a companion diagnostic test to determine patient treatment eligibility. Therefore, demand for precision testing has increased in both laboratories and hospitals.

In 2018, Tsao et al. investigated the interchangeability of some PD-L1 antibodies (20). Among the five antibody assays (22C3, 28-8, SP142, SP264, and 73-10) they evaluated, three antibodies (22C3, 28-8, and SP263) were found to be compatible with one another for determining TPS of primary lung cancer cells. Furthermore, Humphries et al. have demonstrated high concordance between the 22C3 and SP263 IHC assays in NSCLC (28). In the future, the use of any one of these interchangeable antibodies is anticipated to aid treatment decision making. However, the best site for evaluating PD-L1 expression in a patient's body remains unidentified.

Heterogeneity is an important issue in the field of 
22C3 primary tumor

22C3 lymph node

28-8 primary tumor

28-8 lymph node
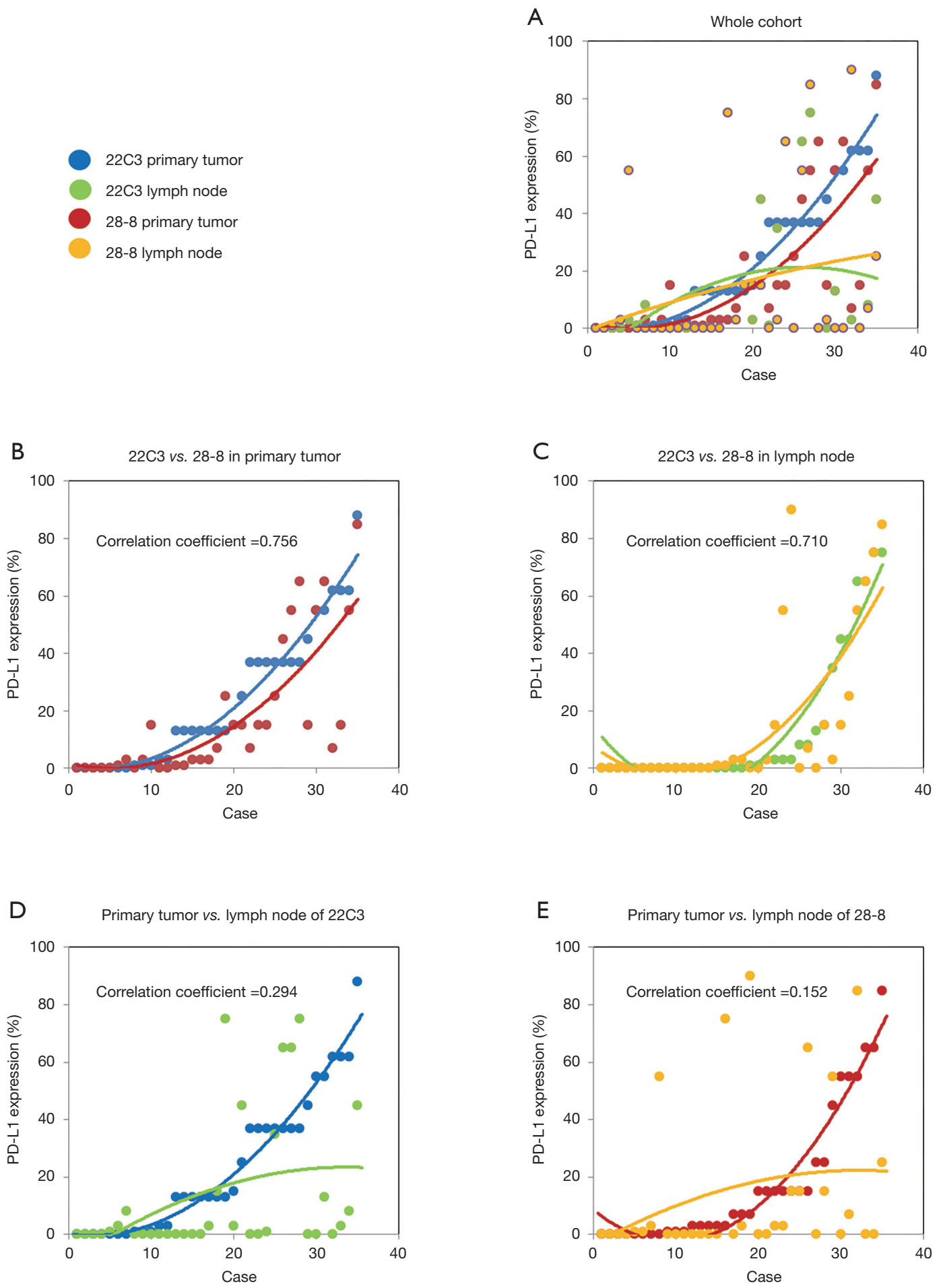

Figure 3 The five scatter plots illustrate the distribution of PD-L1 expression. (A) Each colored dot represents PD-L1 expression; blue (primary tumors stained by 22C3), green (lymph nodes stained by 22C3), red (primary tumors stained by 28-8), and yellow (lymph nodes stained by 28-8); (B) the scatter plot illustrates PD-L1 expression distribution in primary tumors stained by either 22C3 or 28-8 antibodies; (C) the scatter plot depicts a similar correlation of PD-L1 expression in lymph nodes between the 22C3 and 28-8 assays; (D) no correlation of PD-L1 expression was demonstrated between primary tumors and lymph nodes when performing the 22C3 assay; (E) similarly, in the 28-8 assay, there was no statistical association of PD-L1 expression between primary tumors and lymph nodes. 

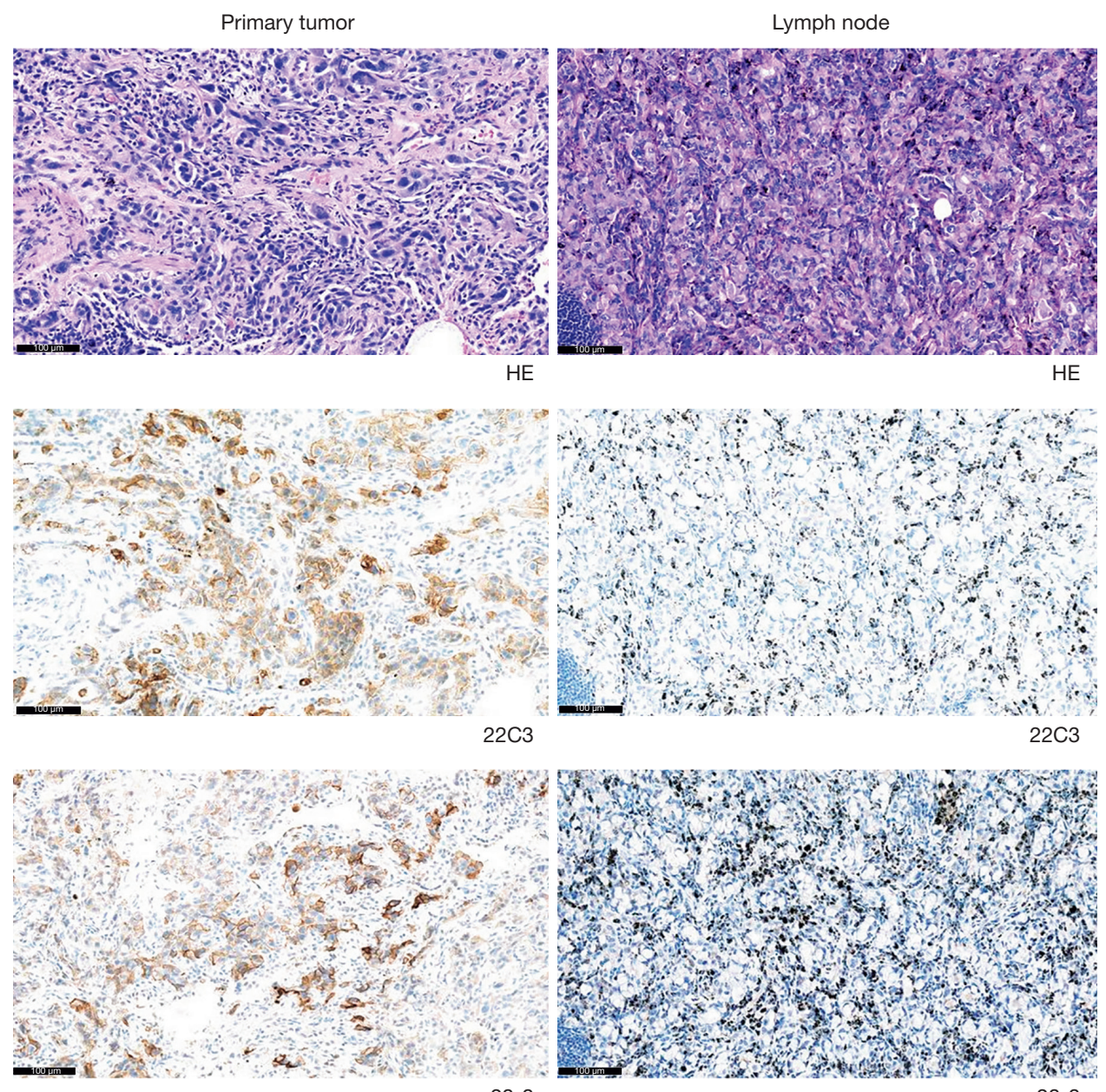

28-8

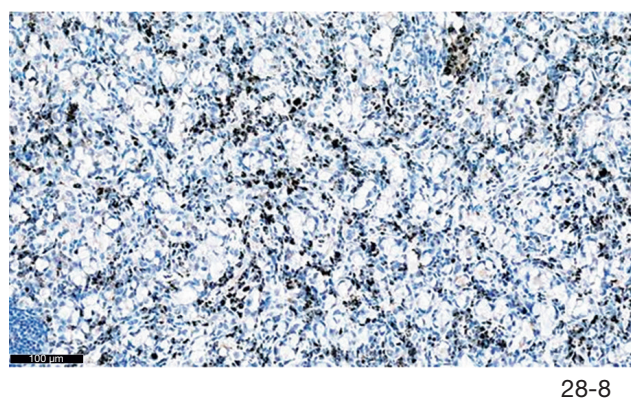

Figure 4 A representative example demonstrating strongly positive staining in primary tumors but no staining in paired metastatic lymph nodes, when using both the 22C3 and 28-8 assays.

oncology, and has been that way for some time (29). Heterogeneity as a concept covers both inter- and intrapatient variability among organs, tissues, cells, and molecules (30). Clinical discussions often involve the site that can be or should be biopsied to determine treatment. Although there are published studies regarding intra-tumor, inter-observer, or inter-assay heterogeneities, there are very few reports on inter-tumor heterogeneity of PD-L1 expression in NSCLC. Sakakibara et al. have reported good concordance of PD-L1 IHC assay between primary tumors and their paired lymph node metastases in NSCLC $(r=0.93$; $\mathrm{P}=0.02)$; however, their patient cohort was very small $(\mathrm{n}=5)$, and non-clinically used antibodies (rabbit monoclonal, clone EPR1161) were implemented in their study (31). We explored differences in PD-L1 expression between primary tumors and their paired lymph node metastases in NSCLC, using the Dako PD-L1 IHC 22C3 and 28-8 pharmDx assays. In our study, while inter-assay heterogeneity was tolerable $(74.2 \%$ in primary tumors and $71.4 \%$ in metastatic lymph nodes), inter-tumor heterogeneity was non-negligible due to the low TPS concordance rate observed $(28.6 \%$ in the $22 \mathrm{C} 3$ assay and $31.4 \%$ in the $28-8$ assay). Results of PD-L1 expression analysis and correlation coefficients were comparable (Figure 3).

According to the current guidelines, PD-L1 staining of tumor cells is a pivotal examination. However, intertumor heterogeneity remains an unsolved clinical problem, which can potentially preclude precise treatment planning for patients with advanced NSCLC. Here, we present a representative case, which may raise controversies among oncologists (Figure 4). In this case, both the 22C 3 and 28-8 IHC assays showed positive staining in primary tumors 
but no staining in paired metastatic lymph nodes. These findings indicate that chemotherapy or monotherapy with pembrolizumab should be recommended for patients with advanced NSCLC when referring to the result of PD-L1 staining in primary tumors. However, these medications should not be administered when lymph node staining is referred. Currently, the ideal site for immunotherapy biomarker remains unknown. This issue must be addressed by future clinical studies that will ideally include a greater number of patients.

This study had several limitations: (I) the relatively small number of cases evaluated; (II) the single center nature of the study; and (III) the surgical resection of all samples. It should be noted that obtaining sufficient specimen volume for PD-L1 IHC assay from both primary tumors and any other metastatic tissues recovered from a patient with or without surgical indications is very difficult in clinical settings. In the future, multicenter prospective studies may elucidate the association between inter-tumor heterogeneity and immunotherapy treatment outcomes.

In summary, we observed a favorable similarity between the detection capabilities of both 22C3 and 28-8 IHC assays and a serious discrepancy in PD-L1 staining between primary and secondary (metastatic) NSCLC tumors. The interchangeability of these two assays should make it easier to select one assay based on cost concerns; however, intertumor heterogeneity may make it difficult to treat patients efficiently. We believe our present study will positively impact future clinical studies regarding the ideal site for testing PD-L1 expression.

\section{Acknowledgments}

We thank Mr. Hidehiro Numagami, Mr. Yasuhito Kobayashi and Mei Miyagawa for excellent technical assistance.

Funding: This work was supported by grants from Saitama Cardiovascular and Respiratory Center (grant numbers: 17ET and 18ET).

\section{Footnote}

Conflicts of Interest: The authors have no conflicts of interests to declare.

Ethical Statement: The authors are accountable for all aspects of the work in ensuring that questions related to the accuracy or integrity of any part of the work are appropriately investigated and resolved. This study was approved by the institutional review board of the Saitama Cardiovascular and Respiratory Center (approval numbers: 2018005). Written informed consent was obtained from all patients to participate in this study, and this study outcome will not affect the future management of the patients.

\section{References}

1. Malvezzi M, Carioli G, Bertuccio P, et al. European cancer mortality predictions for the year 2017, with focus on lung cancer. Ann Oncol 2017;28:1117-23.

2. Siegel RL, Miller KD, Jemal A. Cancer statistics, 2016. CA Cancer J Clin 2016;66:7-30.

3. Hori M, Matsuda T, Shibata A, et al. Cancer incidence and incidence rates in Japan in 2009: A study of 32 populationbased cancer registries for the Monitoring of Cancer Incidence in Japan (MCIJ) project. Jpn J Clin Oncol 2015;45:884-91.

4. Pilkington G, Boland A, Brown T, et al. A systematic review of the clinical effectiveness of fi rst-line chemotherapy for adult patients with locally advanced or metastatic non-small cell lung cancer. Thorax 2015;70:359-67.

5. Nishimura H, Okazaki T, Tanaka Y, et al. Autoimmune Dilated Cardiomyopathy in PD-1 Receptor - Deficient Mice. Science 2001;291:319-22.

6. Iwai $Y$, Ishida $M$, Tanaka $Y$, et al. Involvement of PD-L1 on tumor cells in the escape from host immune system and tumor immunotherapy by PD-L1 blockade. Proc Natl Acad Sci 2002;99:12293-7.

7. Leach DR, Krummel MF, Allison JP. Enhancement of Antitumor Immunity by CTLA-4 blockade. Science 1996;271:1734-6.

8. Ishida Y, Agata Y, Shibahara K, et al. Induced expression of PD-1, a novel member of the immunoglobulin gene superfamily, upon programmed cell death. EMBO J 1992;11:3887-95.

9. Okazaki T, Honjo T. The PD-1-PD-L pathway in immunological tolerance. Trends Immunol 2006;27:195-201.

10. Herbst RS, Baas P, Kim DW, et al. Pembrolizumab versus docetaxel for previously treated, PD-L1-positive, advanced non-small-cell lung cancer (KEYNOTE-010): A randomised controlled trial. Lancet 2016;387:1540-50.

11. Brahmer JR, Rodríguez-Abreu D, Robinson AG, et al. Health-related quality-of-life results for pembrolizumab versus chemotherapy in advanced, PD-L1-positive NSCLC (KEYNOTE-024): a multicentre, international, 
randomised, open-label phase 3 trial. Lancet Oncol 2017;18:1600-9.

12. Vokes EE, Ready N, Felip E, et al. Nivolumab versus docetaxel in previously treated advanced non-small-cell lung cancer (CheckMate 017 and CheckMate 057): 3-year update and outcomes in patients with liver metastases. Ann Oncol 2018;29:959-65.

13. Rittmeyer A, Barlesi F, Waterkamp D, et al. Atezolizumab versus docetaxel in patients with previously treated non-small-cell lung cancer (OAK): a phase 3, openlabel, multicentre randomised controlled trial. Lancet 2017;389:255-65.

14. The Japan Lung Cancer Society. Guidelines for Diagnosis ans Treatment of the Lung Cancer 2018. Available online: https://www.haigan.gr.jp/modules/guideline/index. php?content_id=3 (28 July 2019, date last accessed).

15. Ettinger DS, Aisner DL, Wood DE, et al. NCCN Guidelines Insights: Non-Small Cell Lung Cancer, Version 5.2018. J Natl Compr Canc Netw 2018;16:807-21.

16. Planchard D, Popat S, Kerr K, et al. Metastatic non-small cell lung cancer: ESMO Clinical Practice Guidelines for diagnosis, treatment and follow-up. Ann Oncol 2019;30:863-70.

17. Brahmer JR, Govindan R, Anders RA, et al. The Society for Immunotherapy of Cancer consensus statement on immunotherapy for the treatment of non-small cell lung cancer (NSCLC). J Immunother Cancer 2018;6:75.

18. Büttner R, Gosney JR, Skov BG, et al. Programmed Death-Ligand 1 Immunohistochemistry Testing: A Review of Analytical Assays and Clinical Implementation in NonSmall-Cell Lung Cancer. J Clin Oncol 2017;35:3867-76.

19. Yu H, Boyle TA, Zhou C. PD-L1 Expression in Lung Cancer. J Thorac Oncol 2016;11:964-75.

20. Tsao MS, Kerr KM, Kockx M, et al. PD-L1 Immunohistochemistry Comparability Study in Real-Life Clinical Samples: Results of Blueprint Phase 2 Project. J Thorac Oncol 2018;13:1302-11.

21. Munari E, Zamboni G, Lunardi G, et al. PD-L1 Expression Heterogeneity in Non-Small Cell Lung Cancer: Defining Criteria for Harmonization between

Cite this article as: Saito Y, Horiuchi S, Morooka H, Ibi T, Takahashi N, Ikeya T, Shimizu Y, Hoshi E. Inter-tumor heterogeneity of PD-L1 expression in non-small cell lung cancer. J Thorac Dis 2019;11(12):4982-4991. doi: 10.21037/ jtd.2019.12.24
Biopsy Specimens and Whole Sections. J Thorac Oncol 2018;13:1113-20.

22. Bassanelli M, Sioletic S, Martini M, et al. Heterogeneity of PD-L1 Expression and Relationship with Biology of NSCLC. Anticancer Res 2018;38:3789-96.

23. Li C, Huang C, Mok TS, et al. Comparison of 22C3 PDL1 Expression between Surgically Resected Specimens and Paired Tissue Microarrays in Non-Small Cell Lung Cancer. J Thorac Oncol 2017;12:1536-43.

24. Goldstraw P, Chansky K, Crowley J, et al. The IASLC lung cancer staging project: Proposals for revision of the TNM stage groupings in the forthcoming (eighth) edition of the TNM Classification for lung cancer. J Thorac Oncol 2016;11:39-51.

25. International Association for the Lung Cancer. IASLC Atlas of PD-L1 Testing in Lung Cancer. Available online: https://www.iaslc.org/Portals/0/iaslc_pd-11_atlas_ mar2018_lo-res.pdf?ver=2019-06-06-153849-143

26. Dako. PD-L1 IHC 22C3 pharmDx: Non-Small Cell Lung Cancer [interpretation manual]. Accessed 28 July 2019. Available online: https://www.agilent.com/cs/library/ usermanuals/public/29158_pd-11-ihc-22C3-pharmdxnsclc-interpretation-manual.pdf

27. Dako/Agilent. PD-L1 IHC 28-8 pharmDx: Interpretation Manual (Non-Squamous Non-Small Cell Lung Cancer). Accessed28 July 2019. Available online: https://www. agilent.com/cs/library/usermanuals/public/29126_pd-1128-8-nsclc-interpretation-manual_row.pdf

28. Humphries MP, McQuaid S, Craig SG, et al. Critical Appraisal of Programmed Death Ligand 1 Reflex Diagnostic Testing: Current Standards and Future Opportunities. J Thorac Oncol 2019;14:45-53.

29. Spremulli EN, Dexter DL. Human tumor cell heterogeneity and metastasis. J Clin Oncol 1983;1:496-509.

30. Allison KH, Sledge GW Jr. Heterogeneity and Cancer. Oncology (Williston Park) 2014;28:772-8.

31. Sakakibara R, Inamura K, Tambo Y, et al. EBUS-TBNA as a Promising Method for the Evaluation of Tumor PD-L1 Expression in Lung Cancer. Clin Lung Cancer 2017;18:527-534.e1. 